\title{
Cancer risk among insulin users: comparing analogues with human insulin in the CARING five-country cohort study
}

\author{
Anna But ${ }^{1}$ - Marie L. De Bruin ${ }^{2,3}$ • Marloes T. Bazelier ${ }^{2}$. Vidar Hjellvik ${ }^{4}$. \\ Morten Andersen ${ }^{5,6,7}$ • Anssi Auvinen ${ }^{8}$. Jakob Starup-Linde ${ }^{9}$. \\ Marjanka K. Schmidt ${ }^{10,11} \cdot$ Kari Furu $^{4}$ - Frank de Vries ${ }^{2,12,13,14}$. \\ ${\text { Øystein } \text { Karlstad }^{4} \cdot \text { Nils Ekström }}^{5}$ - Jari Haukka ${ }^{1}$
}

Received: 13 December 2016 / Accepted: 24 April 2017 /Published online: 1 June 2017

(C) The Author(s) 2017. This article is an open access publication

\begin{abstract}
Aims/hypothesis The aim of this work was to investigate the relationship between use of certain insulins and risk for cancer, when addressing the limitations and biases involved in previous studies.

Methods National Health Registries from Denmark (1996-2010), Finland (1996-2011), Norway (2005-2010) and Sweden (2007-2012) and the UK Clinical Practice Research Datalink database (1987-2013) were used to conduct a cohort study on new insulin users $(N=327,112)$. By using a common data model and semi-aggregate approach, we pooled individual-level records from five cohorts and applied Poisson
\end{abstract}

Electronic supplementary material The online version of this article (doi:10.1007/s00125-017-4312-5) contains peer-reviewed but unedited supplementary material, which is available to authorised users.

Anna But

anna.but@helsinki.fi

Marie L. De Bruin

M.L.deBruin@uu.nl

1 Department of Public Health Clinicum, University of Helsinki, Tukholmankatu 8B, P.O. Box 20, 00014 Helsinki, Finland

2 Division of Pharmacoepidemiology and Clinical Pharmacology, Utrecht Institute for Pharmaceutical Sciences, Utrecht University, Universiteitsweg 99, 3584 CG Utrecht, the Netherlands

3 Copenhagen Centre for Regulatory Science (CORS), Department of Pharmacy, University of Copenhagen, Copenhagen, Denmark

4 Department of Pharmacoepidemiology, Norwegian Institute of Public Health, Oslo, Norway

5 Centre for Pharmacoepidemiology, Karolinska Institutet, Stockholm, Sweden

6 Research Unit of General Practice, University of Southern Denmark, Odense, Denmark regression models. For each of ten cancer sites studied, we estimated the rate ratios (RRs) by duration $(\leq 0.5,0.5-1,1-2$, $2-3,3-4,4-5,5-6$ and $>6$ years) of cumulative exposure to insulin glargine or insulin detemir relative to that of human insulin.

Results A total of 21,390 cancer cases occurred during a mean follow-up of 4.6 years. No trend with cumulative treatment time for insulin glargine relative to human insulin was observed in risk for any of the ten studied cancer types. Of the 136 associations tested in the main analysis, only a few increased and decreased risks were found: among women, a higher risk was observed for colorectal (RR 1.54, 95\% CI 1.06, 2.25) and
7 Department of Drug Design and Pharmacology, University of Copenhagen, Copenhagen, Denmark

8 Department of Epidemiology, School of Health Sciences, University of Tampere, Tampere, Finland

9 Department of Endocrinology and Internal Medicine, Aarhus University Hospital THG, Aarhus, Denmark

10 Division of Molecular Pathology, The Netherlands Cancer Institute Antoni van Leeuwenhoek Hospital, Amsterdam, the Netherlands

11 Division of Psychosocial Research and Epidemiology, The Netherlands Cancer Institute - Antoni van Leeuwenhoek Hospital, Amsterdam, the Netherlands

12 The Netherlands Department of Clinical Pharmacy and Toxicology, Mastricht University Medical Centre, Maastricht, the Netherlands

13 The Netherlands Research Institute CAPHRI, Maastricht University, Maastricht, the Netherlands

14 The Netherlands MRC Lifecourse Epidemiology Unit, University of Southampton, Southampton, UK 
endometrial cancer (RR 1.78, 95\% CI 1.07, 2.94) for $\leq 0.5$ years of treatment and for malignant melanoma for $2-3$ years (RR $1.92,95 \%$ CI $1.02,3.61$ ) and $4-5$ years (RR $3.55,95 \%$ CI 1.68 , 7.47]); among men, a lower risk was observed for pancreatic cancer for 2-3 years (RR 0.34, 95\% CI 0.17, 0.66) and for liver cancer for 3-4 years (RR $0.36,95 \%$ CI $0.14,0.94$ ) and $>6$ years (RR 0.22, 95\% CI 0.05, 0.92). Comparisons of insulin detemir with human insulin also showed no consistent differences.

Conclusions/interpretation The present multi-country study found no evidence of consistent differences in risk for ten cancers for insulin glargine or insulin detemir use compared with human insulin, at follow-up exceeding 5 years.

Keywords Cancerrisk - Cohort study - Common data model . Cumulative treatment time - Detemir - Glargine $\cdot$ Human insulin $\cdot$ Insulin analogues $\cdot$ New insulin user · Poisson model · Rate ratio $\cdot$ Semi-aggregate $\cdot$ Site-specific

$\begin{array}{ll}\text { Abbreviations } \\ \text { ATC } & \text { Anatomical Therapeutic Chemical } \\ \text { CARING } & \text { Cancer risk and insulin analogues } \\ \text { CPRD } & \text { Clinical Practice Research Datalink } \\ \text { DDD } & \text { Defined daily dose } \\ \text { HRT } & \text { Hormone replacement treatment } \\ \text { NIAD } & \text { Non-insulin antidiabetic drug } \\ \text { NSAID } & \text { Nonsteroidal anti-inflammatory drug } \\ \text { RR } & \text { Rate ratio }\end{array}$

\section{Introduction}

Diabetes mellitus and cancer are common diseases with rising incidence and prevalence globally [1,2]. Diabetes is associated with an increased risk for certain cancers [3] and the pattern and magnitude of the excess risk are generally similar for type 1 and 2 diabetes $[4,5]$. It has been suggested that certain diabetes risk factors, as well as glucose-lowering medications, may contribute to this association [6]. In 2009, the publication of four observational studies [7-10] sparked concerns about insulin glargine as a potential modifier of cancer risk [11].

The oncogenic potential of various insulin analogues has been suggested by preclinical safety evaluations showing that IGF and insulin receptor signalling pathways, which are essential for mitogenic potency, are affected by ligand-specific receptor dynamics, depending on the cell type [12]. Initial observational studies [7-10] were criticised for limitations and biases $[11,13,14]$ such as short follow-up, inclusion of prevalent insulin users and time-lag bias [15]. Further attempts to rule out or confirm the association yielded inconsistent findings, emphasising the importance of properly designed and conducted observational studies [16].
The evidence from the observational studies remains inconsistent $[17,18]$, particularly due to the involvement of methodological drawbacks, such as time-related biases and selection bias [15]. Moreover, the findings from studies that ignore doseeffect aspects, time-varying character of exposure, site-specific risks or long latency of cancer are limited in their biological plausibility $[16,19]$. It was thought that RCTs, the gold standard of treatment comparisons, would disentangle the association. However, the ORIGIN trial, which showed a null effect [20] and constitutes perhaps the strongest RCT evidence concerning the use of insulin analogues and cancer outcomes, has been criticised for evaluation of cancer risk with respect to the initial insulin glargine allocation but not the cumulative insulin glargine use [21]. Overall, there is little robust evidence from observational studies or RCTs to draw firm conclusions.

The present observational study, which is a part of the Cancer Risk and Insulin Analogues (CARING) project, sought to investigate the effect of exposure to insulin glargine or insulin detemir on cancer risk as compared with that of human insulin, mitigating through study design and analytical approaches the limitations and biases involved in the previous studies.

\section{Methods}

Overview This cohort study on new insulin users was conducted using nationwide data from the Norwegian, Swedish, Danish and Finnish National Health Registries, as well as data from UK general practitioners in the Clinical Practice Research Datalink (CPRD). Project partners obtained ethics approval from their respective authorities. The study protocol, where data sources and study cohorts are described in more detail, was registered in the European Network of Centres for Pharmacoepidemiology and Pharmacovigilance (ENCePP) electronic register of studies [22]. To synchronise definitions of demographics, exposures, outcomes and confounders, a common data model and concept dictionary were developed. Table 1 shows the study design and methodological approaches to the data analysis that we implemented to mitigate different types of selection, information and time-related biases often inherent in the observational research $[15,23-25]$.

Data sources, selection and follow-up of participants National health registries in the Nordic countries comprise computerised records for the entire population of 26 million people, each of whom are assigned a unique personal identification number. The cancer registries have a long tradition of providing comparable and high-quality data with almost $100 \%$ coverage of incident cancer cases [26]. Prescription registries, established in 1995 in Denmark and Finland, in 2004 in Norway and in 2005 in Sweden, have provided ample data for pharmaco-epidemiological research [27]. The CPRD, 


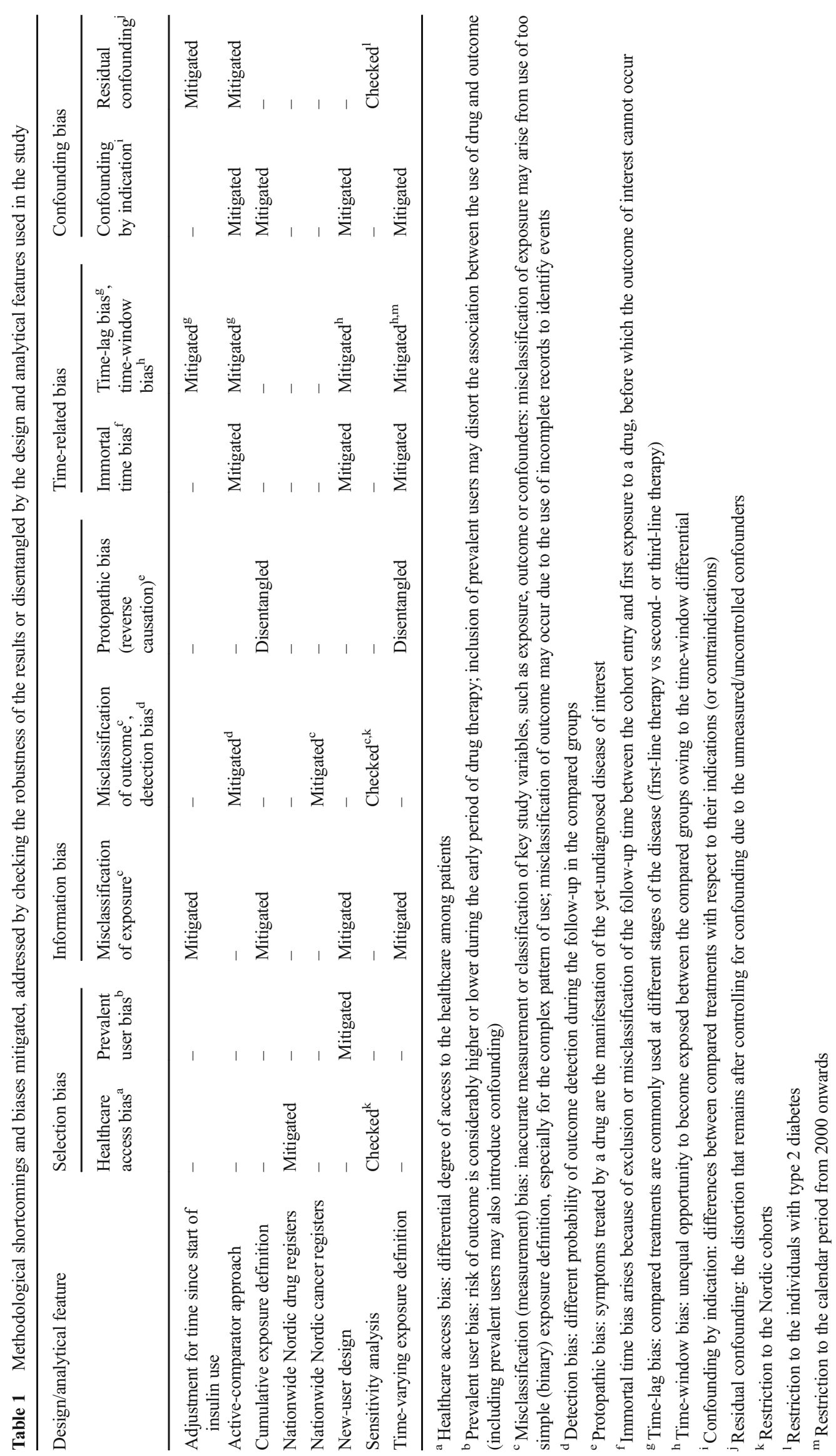


a large computerised database established in the UK in 1987 , contains anonymised medical records, including demographics, prescriptions and cancer diagnoses, that are considered to be of good quality [28]. Currently, 4.4 million individuals, $6.9 \%$ of the UK population, meet the quality criteria and are broadly representative of the entire population with regard to demographic characteristics [29].

Within the study period (Table 2) defined as the period of valid data collection [22], we identified all individuals having at least one insulin purchase (Nordic countries) or prescription (CPRD). Nordic cohorts were linked with the registered data on cancer, death and emigration; data for the British cohort were compiled from information on cancer and death available from the CPRD. New insulin users, who were defined based on a 1 year lead-in period, were included if they had no history of cancer (except non-melanoma skin cancer) and were aged $\geq 18$ years on the first prescription for any insulin (index date). Follow-up started at index date and ended at the date of emigration (Sweden, Denmark and Norway) or transfer out of the CPRD, diagnosis of any cancer (excluding nonmelanoma skin cancer), death or end of follow-up, whichever occurred first.

Cancer outcomes, insulin treatments and potential confounders We relied on coding dictionaries, compiled according to different coding systems (ICD-7, ICD-9 [www. icd9data.com/2007/Volume1], ICD-10 [www.who.int/ classifications/icd/en/] and ICD-O-3 in the Nordic countries; Read code system in the CPRD), to identify incident cancer cases defined as the first occurrence of any cancer type [22]. Multiple cancers diagnosed on the same date were considered as distinct site-specific endpoints. Our primary interest was in

Table 2 Baseline and follow-up characteristics of the study cohorts of new users of insulin

\begin{tabular}{|c|c|c|c|c|c|}
\hline Characteristic & $\begin{array}{l}\text { Denmark } \\
(N=66,698)\end{array}$ & $\begin{array}{l}\text { Finland } \\
(N=105,945)\end{array}$ & $\begin{array}{l}\text { Norway } \\
(N=21,541)\end{array}$ & $\begin{array}{l}\text { Sweden } \\
(N=85,319)\end{array}$ & $\begin{array}{l}\mathrm{UK}(\mathrm{CPRD}) \\
(N=47,609)\end{array}$ \\
\hline Study period ${ }^{\mathrm{a}}$ & 1996-2010 & $1996-2011$ & 2005-2010 & 2007-2012 & $1987-2013$ \\
\hline Male sex, $n(\%)$ & $38,292(57)$ & $57,691(55)$ & $12,053(56)$ & $48,931(57)$ & $25,589(54)$ \\
\hline Age years, mean $(\mathrm{SD})^{\mathrm{b}}$ & $60.1(16.0)$ & $61.6(15.9)$ & $57.3(17.8)$ & $64.0(16.4)$ & $59.3(16.3)$ \\
\hline \multicolumn{6}{|l|}{ Age years, $n(\%)^{\mathrm{b}}$} \\
\hline $18-30$ & $3193(4.8)$ & $4481(4.2)$ & $1634(7.6)$ & $3121(3.6)$ & $2555(5.4)$ \\
\hline $30-40$ & $5075(7.6)$ & $7317(6.9)$ & $2653(12.3)$ & $4924(5.8)$ & $4540(9.5)$ \\
\hline $40-50$ & $8334(12.5)$ & $11,095(10.5)$ & $3088(14.3)$ & $8383(9.8)$ & $5825(12.2)$ \\
\hline $50-60$ & $14,432(21.6)$ & $22,928(21.6)$ & 4248 (19.7) & $14,870(17.4)$ & $9351(19.6)$ \\
\hline $60-70$ & $16,306(24.5)$ & $25,254(23.8)$ & $4220(19.6)$ & $21,378(25.1)$ & $11,661(24.5)$ \\
\hline $70-80$ & $12,527(18.8)$ & $22,110(20.9)$ & $3194(14.8)$ & $17,176(20.1)$ & $9505(20.0)$ \\
\hline $80+$ & $6831(10.2)$ & $12,760(12.0)$ & 2504 (11.6) & $15,466(18.1)$ & $4172(8.8)$ \\
\hline \multicolumn{6}{|l|}{ Follow-up time, years } \\
\hline Mean (SD) & $5.3(3.9)$ & $5.6(3.9)$ & $2.7(1.8)$ & $2.7(1.8)$ & $5.7(4.3)$ \\
\hline Median (interquartile range) & $4.5(1.9,7.8)$ & $4.7(2.3,8.3)$ & $2.5(1.1,4.1)$ & $2.6(1.1,4.1)$ & $4.7(1.9,8.4)$ \\
\hline $\begin{array}{l}\text { No. of person-years/1000, } \\
\text { all (male sex) }\end{array}$ & $331.2(184.4)$ & $589.1(316.9)$ & $57.8(32.4)$ & $226.6(131.3)$ & $265.3(141.6)$ \\
\hline \multicolumn{6}{|l|}{ Ever-use, $n(\%)^{\mathrm{c}}$} \\
\hline Human insulin & $54,216(81)$ & $68,894(65)$ & $17,579(82)$ & $48,976(57)$ & $23,183(49)$ \\
\hline Insulin glargine & $7151(11)$ & $43,741(41)$ & $1447(7)$ & $15,138(18)$ & $15,374(32)$ \\
\hline Insulin detemir & $9520(14)$ & $24,593(23)$ & $868(4)$ & $4367(5)$ & $7373(15)$ \\
\hline Other insulin & $33,388(50)$ & $48,280(46)$ & $14,376(67)$ & $53,810(63)$ & $27,491(58)$ \\
\hline \multicolumn{6}{|l|}{ Baseline use, $n(\%)$} \\
\hline $\mathrm{HRT}^{\mathrm{d}}$ & $5187(18)$ & $6546(14)$ & $1641(17)$ & $6621(18)$ & $1530(7)$ \\
\hline NSAID $^{\mathrm{e}}$ & $17,800(27)$ & $29,609(28)$ & $5437(25)$ & $16,485(19)$ & $8935(18)$ \\
\hline Any oral glucose-lowering therapy & $49,569(74)$ & $83,935(79)$ & $15,051(70)$ & $62,522(73)$ & $37,239(78)$ \\
\hline Statin & $22,948(34)$ & $38,493(36)$ & $9309(43)$ & $39,635(46)$ & $24,188(51)$ \\
\hline
\end{tabular}

${ }^{a}$ Start of study period defined according to the start of prescription registry (Nordic countries) or start of valid data collection (CPRD)

${ }^{\mathrm{b}}$ Age at baseline

${ }^{\mathrm{c}}$ Ever-use of specific insulin during the follow-up

${ }^{\mathrm{d}}$ Female sex only

${ }^{\mathrm{e}}$ Based on prescriptions only 
ten site-specific cancers. Based on NORDCAN data (cancer statistics from Nordic countries) [26], we selected the eight cancer types with the highest incidence rates (ICD-10 codes): trachea and lung (C33, C34), melanoma of skin (C43), bladder (C67), colorectal (C18-21), non-Hodgkin lymphoma (C82-86, C88.4), breast (C50), endometrial (C54) and prostate (C61). Liver (C22) and pancreatic (C25) cancers were also included because of their strong association with diabetes. As a secondary outcome of interest, we investigated the first occurrence of any cancer.

Based on Anatomical Therapeutic Chemical (ATC) classification codes [30] (British National Formulation codes for the CPRD), we identified users of human insulin (A10AC01, A10AB01, A10AD01, A10AE01, A10AF01) and the insulin analogues insulin glargine (A10AE04) and insulin detemir (A10AE05). Any other insulins and analogues were considered as a single group. Prescription data form the Nordic registries included the date and amount purchased, in defined daily doses (DDDs) [30], but no information on individual dosage. For the CPRD cohort, we derived DDDs from the dosage information (substance strength and amount) contained within prescription data. We assumed a daily consumption of 1 DDD per day and transformed each drug record into a period covered by the number of DDDs.

For each insulin type of interest, we defined insulin exposure time-dependently as a cumulative treatment time. After splitting the individual follow-up period into intervals of 120 days, the exposure at the beginning of each interval was updated. The exposure began on the date of first prescription/ purchase, after which point an individual was considered exposed. Cumulative treatment time accrued until exposure stopped and remained unchanged, unless treatment was resumed (see electronic supplementary material [ESM] Methods). We then divided cumulative treatment time into half-year categories for the first year and 1 year categories for longer exposure; the last categories were $>6$ years for the broadly categorised exposure and 9-10 years for the finely categorised exposure. In addition, each exposure variable incorporated a non-exposed category assigned to individuals remaining unexposed to the specific insulin.

We considered only confounders available in all five datasets [22]. In addition to age, sex and calendar time, this included use of non-insulin glucose-lowering drugs (ATC code A10B), statins (C10A), nonsteroidal anti-inflammatory drugs (NSAIDs; M01A) and hormone replacement treatment (HRT; G03), defined as at least one prescription within 1 year before the index date. We also derived several other potential confounders: type 1 diabetes mellitus was assigned to those aged $\leq 30$ years with no non-insulin glucose-lowering drug on the index date; type 2 diabetes mellitus was assigned to those aged $\geq 40$ years with or without non-insulin glucose-lowering drugs; unspecified diabetes type was assigned to the rest of the cohort. We specified the duration of insulin-treated diabetes as time since the index date (in 1 year intervals) and defined menopausal status time-dependently based on cut-off of 50 years of age. Furthermore, the country of the data origin served as a covariate.

Statistical methods The individual-level data from the five cohorts were standardised by each research partner locally using the common data model. We then conducted centralised analyses by uploading the unified data to a server at Statistics Denmark, where for each cohort we constructed the individuallevel dataset to assess insulin exposure and other variables in exactly the same way. We employed a semi-aggregate level approach [31] to combine the datasets, which were tabulated by cancer site as the number of cancer cases and person-years aggregated by categorical variables. To estimate the incidence rates, we fitted multivariable Poisson regression models to the event numbers with the natural log of person-years as an offset. Each model included all three time-dependent insulin exposure variables and was adjusted for time-dependent age and duration of insulin-treated diabetes, sex (not in the sex-stratified analysis), baseline calendar time, use of non-insulin antidiabetic drugs (NIADs), other co-medication (when relevant [22]) and country.

We conducted an active-comparator analysis [25], where the drug of interest is compared with another drug commonly used for the same indication rather than with no treatment. Inclusion of all three insulin exposures in the same model allowed us to calculate the rate ratios (RRs) and 95\% CIs for a particular exposure category by contrasting the incidence rates, which were estimated for each insulin type and duration. In the primary analyses, we examined sex- and site-specific cancer endpoints without separating between diabetes types and using insulin exposures with a broader category ( $>6$ years) for the longer cumulative treatment time. For the secondary analyses, we performed similar evaluations without stratifying on sex and using insulin exposures with finer categories.

Sensitivity analysis We also performed several sensitivity analyses. We restricted the analyses to those who met type 2 diabetes criteria to check whether the results change by diabetes type (data for individuals with type 1 diabetes were limited). In Europe, marketing authorisation for the long-acting insulin analogues insulin glargine and insulin detemir was granted in June 2000 and June 2004, respectively [32, 33]. Coincidentally, usage of two already-approved rapid-acting insulin analogues, insulin lispro and insulin aspart, gained popularity in the early 2000 s. To account for the changes in the profiles of new insulin users, we excluded those entered before 2000. By excluding the CPRD, we addressed the potential of underestimating cancer incidence due to case ascertainment through the CPRD only without linkage to the national cancer registration data. For breast and endometrial cancer, we further adjusted for menopausal status. 
We used version 3.2.2 of R (www.R-project.org) [34] to perform all statistical analyses, the Epi package, version 1.1. 71 (https://cran.r-project.org/web/packages/Epi/index.html) [35] to carry out exposure calculations and the forestplot package, version 1.7 (https://cran.r-project.org/web/ packages/forestplot/index.html) [36] for the graphical output.

\section{Results}

In the five cohorts totalling 327,112 new insulin users, men predominated and the mean age at baseline varied between 57 and 64 years (Table 2). For the combined data, the mean follow-up time was 4.6 years (median 3.7, interquartile range 1.7-6.3). At the end of follow-up, there was $212,848,82,851$ and 46,721 ever-users of human insulin, insulin glargine and insulin detemir, respectively. In all cohorts, human insulin predominated in ever-use patterns. Ever-use of insulin glargine and insulin detemir was most common in the Finnish cohort, as was the baseline use of non-insulin glucose-lowering therapy. Baseline use of other medication also differed between the cohorts.

A total of 1.47 million person-years accumulated and 21,390 new cancer cases occurred during the follow-up. Table 3 shows country- and sex-specific crude incidence rates for the ten site-specific cancers and any cancer. Prostate cancer in men and breast cancer in women showed the highest incidence rates in all cohorts except the Norwegian, where pancreatic cancer was the most common cancer. About $32 \%$ of all cancer cases and the majority of pancreatic cancer cases (63\%) were diagnosed during the first year of insulin treatment.

The results of the sex- and site-specific analyses showed no systematic differences across sites and exposure categories (Figs 1, 2 and ESM Table 1). In women, a higher risk for colorectal (RR 1.54, 95\% CI 1.06, 2.25) and endometrial (RR 1.78, 95\% CI 1.07, 2.94) cancer was observed for the first half-year of cumulative treatment time on insulin glargine relative to that on human insulin and a higher risk for melanoma of skin for $2-3$ years (RR 1.92, 95\% CI 1.02, 3.61) and 4-5 years (RR 3.55, 95\% CI 1.68, 7.47). In men, similar comparisons yielded a lower risk for pancreatic cancer for 2-3 years of exposure (RR $0.34,95 \%$ CI $0.17,0.66$ ), and for liver cancer for $3-4$ years (RR $0.36,95 \%$ CI $0.14,0.94$ ) and $>6$ years (RR $0.22,95 \%$ CI $0.05,0.92$ ). In addition, the results suggested an increase in the risk for bladder (RR 1.41, $95 \%$ CI $0.92,2.17$ ) and colorectal cancer (RR $1.28,95 \%$ CI $0.94,1.75)$ in men for $<0.5$ years and for breast cancer for $<0.5$ years (RR $1.32,95 \%$ CI $0.98,1.79$ ) and $0.5-1$ years (RR1.32, 95\% CI 0.95, 1.85) in women.

In similar analyses performed using the 1 year categories for longer duration of exposure ( $\geq 6$ years) and both sexes combined (not sex-specific cancers), results remained similar (ESM Fig. 1): RR (95\% CI) $1.41(1.11,1.79)$ for colorectal cancer for $<0.5$ year, $0.67(0.43,1.03)$ for pancreatic cancer for 2-3 years, $0.44(0.21,0.91)$ for liver cancer for 3-4 years and $1.60(1.05,2.43)$ for melanoma of the skin for $4-5$ years of insulin glargine vs human insulin use. Comparisons of insulin detemir vs human insulin and insulin glargine vs insulin detemir also showed no consistent differences in sexand site-specific incidence rates (ESM Table 1) as well as in the analyses combining both sexes (ESM Figs 2 and 3).

For any cancer in women, we found an elevated risk for 0.5 year of insulin glargine use relative to human insulin (RR $1.17,95 \%$ CI 1.03, 1.32); in men, there was a lower risk for $0.5-1$ year (RR $0.87,95 \%$ CI $0.77,1.00$ ), $1-2$ years (RR 0.84 , $95 \% \mathrm{CI} 0.73,0.95$ ) and $>6$ years (RR $0.61,95 \% \mathrm{CI} 0.48,0.78$ ) of exposure (Figs 1, 2 and ESM Table 1). Analysis performed without stratifying on sex (Fig. 3) yielded an elevated risk for any cancer for insulin glargine use relative to human insulin for 0.5 year (RR $1.12,95 \%$ CI 1.03, 1.20) and a lower risk for 1-2 years (RR $0.90,95 \%$ CI $0.83,0.98$ ), 6-7 years (RR 0.72 , $95 \%$ CI $0.56,0.91$ ) and $7-8$ years (RR $0.62,95 \%$ CI 0.44 , $0.86)$. Other analyses yielded a lower risk for any cancer in men for $0.5-1,2-3$ and $>6$ years of insulin detemir use relative to that of human insulin (ESM Table 1), and an increased risk in men and women combined (RR 1.18, 95\% CI 1.05, 1.33) for $<0.5$ years of insulin glargine use relative to that of insulin detemir (Fig. 3).

Results were robust across a range of sensitivity analyses (ESM Tables 2, 3). Inclusion of the population with type 2 diabetes (1.31 million person-years [90\%], 21,151cancer cases [99\%]) yielded similar results to those of the primary analysis. The results did not change after either restriction of the study period to the time after insulin glargine's launch (1.12 million person-years [77\%], 16,838 cancer cases [79\%]) or restriction of the study population to the Nordic cohorts. For breast and endometrial cancers, the results remained unchanged after further adjustment for menopausal status.

\section{Discussion}

In the cohort study of 327,112 new insulin users from five European countries, we addressed the relationship between insulin use and cancer incidence of ten site-specific cancers and any cancer, when mitigating methodological shortcomings and biases involved in previous studies. Comparisons of cancer incidence by cumulative treatment time using active comparators showed no consistent differences in the cancer risk for insulin glargine or insulin detemir use relative to that of human insulin use. Although we observed increased and decreased cancer risks for some sites and treatment durations, no trends in the risk with duration of use were seen.

The findings of previous observational studies on the relationship between cancer risk and use of insulin glargine are 


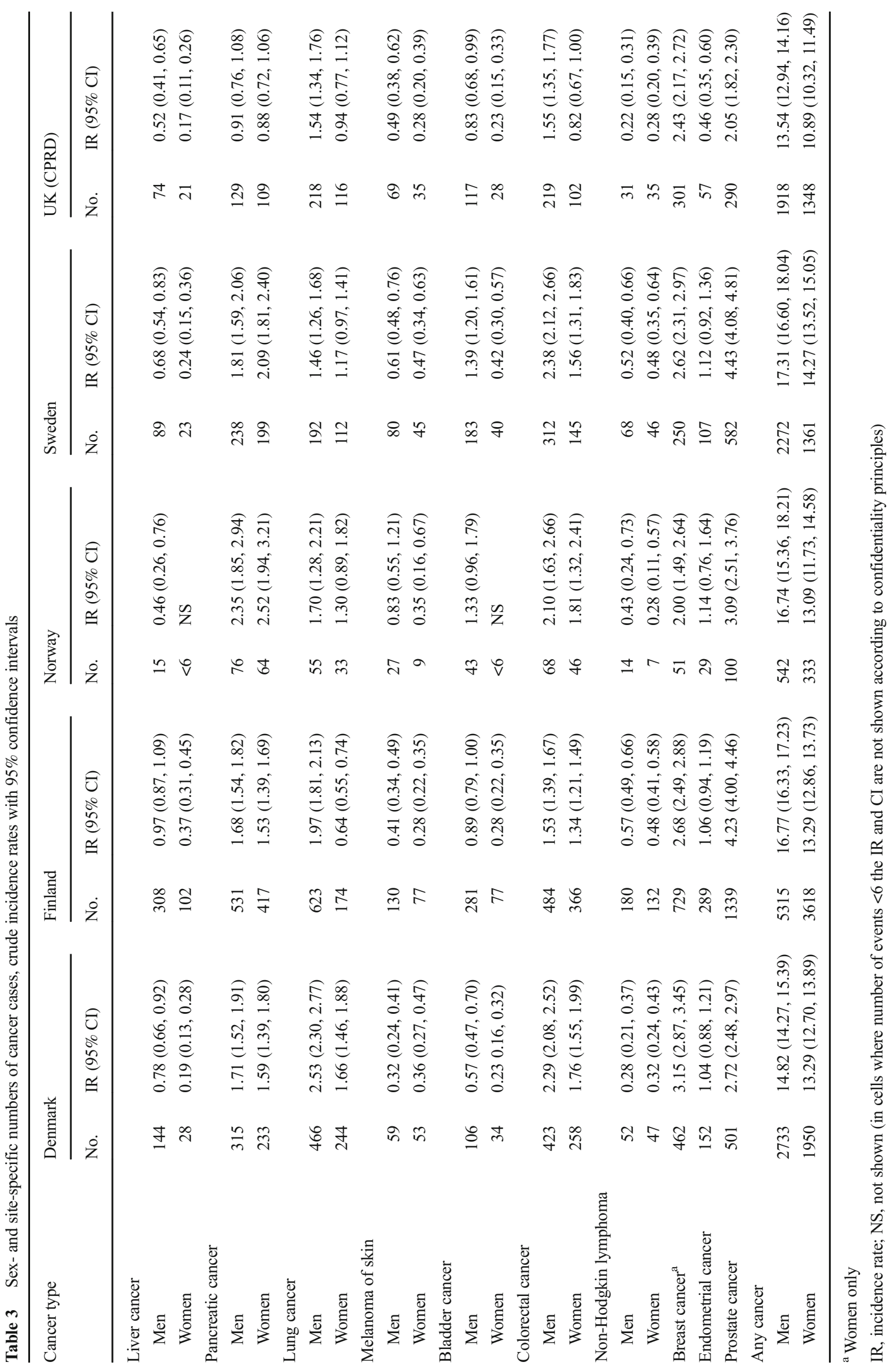


Fig. 1 RR (adjusted for age, calendar time, NIADs, duration of insulin-treated diabetes, country; for liver and colorectal cancers, additional adjustment for relevant co-medications) with $95 \%$ CI for site-specific cancers and any cancer in men by cumulative treatment time (years) on insulin glargine vs human insulin

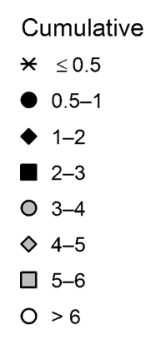

Cumulative
$* \leq 0.5$
$-0.5-1$
$-1-2$
$\square 2-3$
$03-4$
$\diamond 4-5$
$\square 5-6$
$0>6$

Liver cancer

Lung cancer

Melanoma of skin

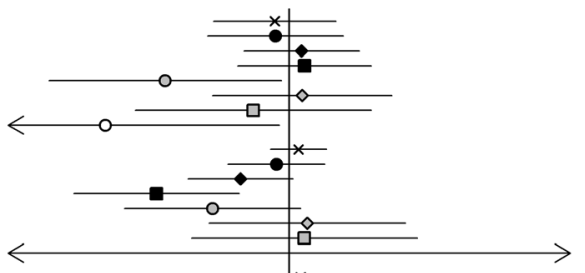

Bladder cancer

Colorectal cancer

Non-Hodgkin lymphoma

Prostate cancer

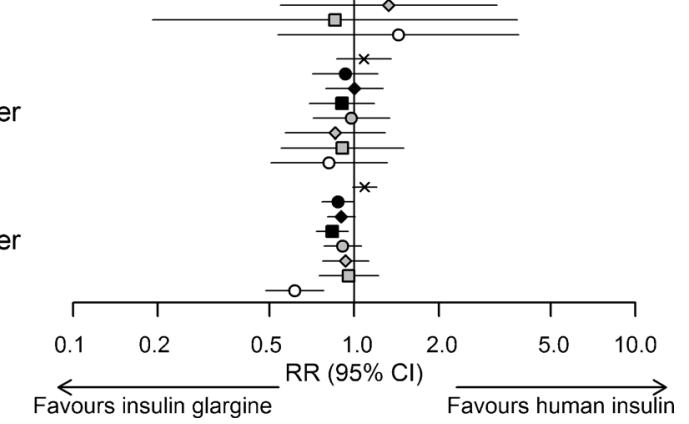

Any cancer

Favours insulin glargine

Favours human insulin

conflicting and may involve methodological limitations and biases $[15,17]$. Most of the current evidence is based on short follow-up and use of an elementary representation of exposure, ignoring the dose or duration of insulin exposure. The latter may result in the inadequate risk estimates, especially when treatment durations of widely differing lengths are considered equivalent $[15,19]$. Of the recent studies with a short follow-up as a main limitation [15], only four were built on a new-user cohort [37-40], and two assessed cancer risk by treatment durations using an active-comparator approach [37, 38].

In the observational study on new users of insulin glargine ( $n=43,306)$ and human insulin $(n=9147)$ enrolled in a US health plan, no association with the risk for prostate, breast, colon and any cancer was found for treatment durations of 0 $6,6-12,12-24$ or $\geq 24$ months [38]. However, the risk estimates reported in this study were imprecise due to the small reference group. An observational study on a cohort of 70,027 new insulin users in France found no differences in risk at median follow-up <3 years for bladder, breast, colorectal, head and neck, liver, lung and kidney cancer between new users of insulin glargine and other basal insulins, when excluding the first year of use and defining exposure as ever-use or cumulative dose [40]. A study of a cohort of 19,337 incident insulin users from the Netherlands found a decreased risk for overall and colon cancer but no difference in risk for bladder, respiratory tract and prostate cancer, when comparing time-dependently defined cumulative time using insulin glargine to that using human insulin, though without further distinction between different treatment durations [39].

In the present study, we found that $22-63 \%$ of cancer cases were diagnosed within the first year after starting insulin treatment. Exclusion of $0.5-1$ year of insulin use, or analysis of short-term use only, may preclude observing the actual dynamics of cancer incidence among insulin users and thus may hinder a better understanding of the nature of the link between diabetes and cancer. For the first half-year of cumulative treatment time on insulin glargine relative to that on human insulin, we found an increased risk for colorectal and any cancer in women and both sexes combined, and for endometrial cancer in women. These findings suggest possible involvement of detection or protopathic bias [41]. The latter is more likely to 
Fig. 2 RR (adjusted for age, calendar time, NIADs, duration of insulin-treated diabetes, country; for liver, colorectal, breast and endometrial cancers, additional adjustment for relevant co-medications) with $95 \% \mathrm{CI}$ for site-specific cancers and any cancer in women by cumulative treatment time (years) on insulin glargine vs human insulin

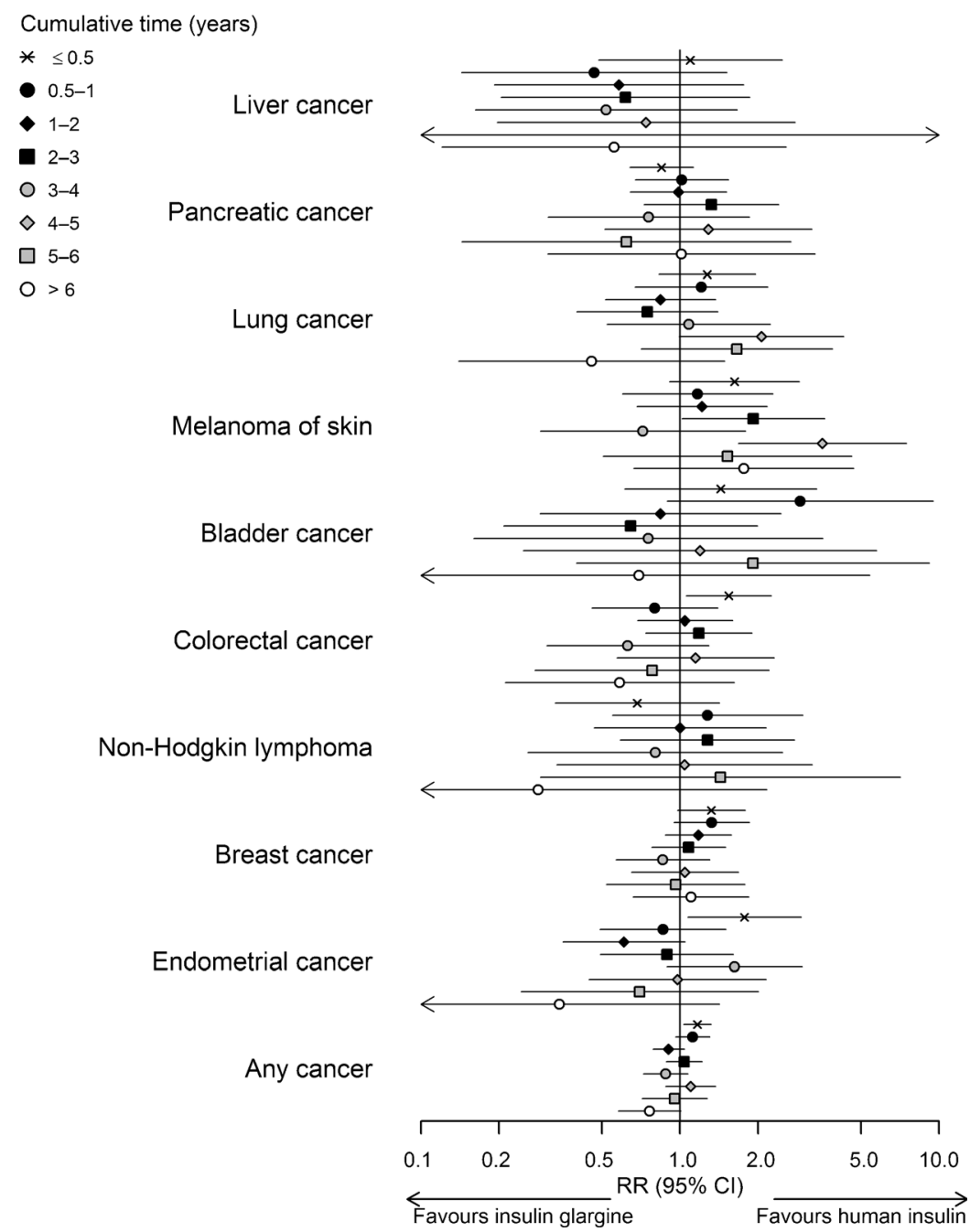

affect the present study, wherein use of insulin glargine was less common than that of human insulin and initiation of insulin glargine was often preceded by use of other insulins, predominantly human insulin. According to the current guidelines, switching from human insulin to insulin glargine should be considered if an individual has hypoglycaemia or fails to reach the target glucose level [42]. Poor glycaemic control may be a sign of underlying cancer and thus switching insulins because of highly variable blood glucose could be associated with more frequently detected cancer.

Although the results of the present study suggest a shift towards increased risk for breast cancer for the initial year of insulin glargine vs human insulin use, no differences were found for longer durations of treatment, when using appropriate comparators and adjusting for the overall time on insulin. In contrast to our study, three recent studies found an association between use of insulin glargine and increased risk for breast cancer [37, 39, 43]. However, those findings may reflect an imbalance in comparator and exposure characteristics rather than differences in cancer risk due to insulin use itself. A study on the UK's General Practice Research Database cohort revealed an elevated risk for $>5$ years since the start of insulin glargine, when comparing insulin glargine users with previous use of insulin vs prevalent users of other insulins, matched on prior insulin duration at baseline [37]. Comparison by time since start of insulin glargine use is likely to provide an unbiased estimate for short-term use but not for longer use where the actual time on insulin and time since the initiation may differ noticeably between comparators. A study from the Netherlands reported an elevated risk for breast cancer when using cumulative durations without differentiation between them, thus ignoring an imbalance between comparators in the follow-up times (median of 2.2 years for insulin glargine users and 3.8 years for human insulin users) [39]. A recent study on a cohort of 12,468 new insulin users from the UK's CPRD also reported an increased risk for breast cancer among new users of insulin glargine with extensive past exposure to other insulins, when comparing insulin glargine use of $>3$ years with ever-use of other insulins [43]. Comparison of specific duration with ever-use may yield a biased result, especially when the time period covered by specific duration differs considerably from the period covered by ever-use. 
Fig. 3 RR (adjusted for age, calendar time, sex, NIADs, duration of insulin-treated diabetes, country) with $95 \% \mathrm{CI}$ for any cancer: pairwise comparisons of insulin glargine, insulin detemir and human insulin by the cumulative treatment time (years)

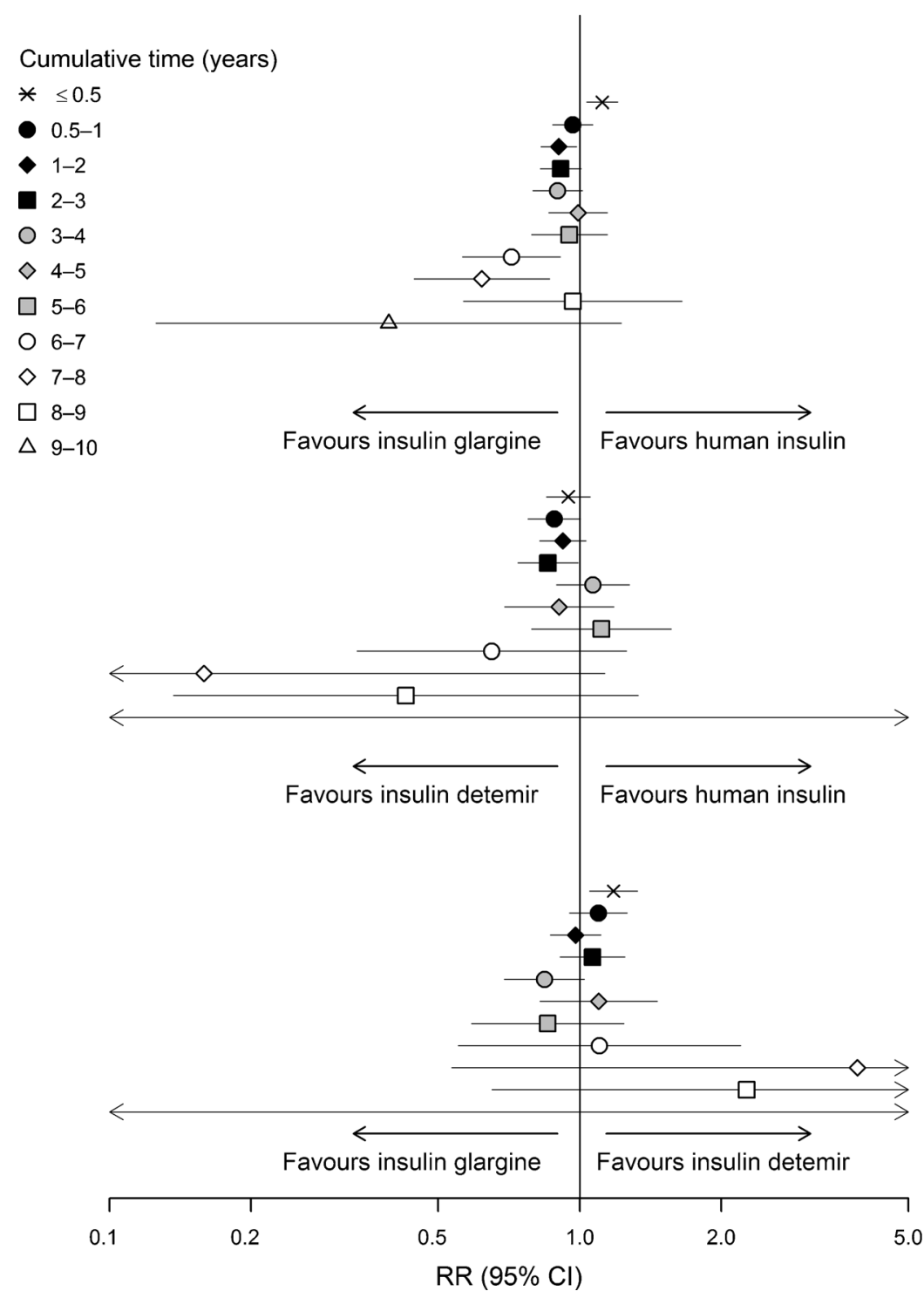

When comparing longer cumulative treatment time on insulin glargine with time on human insulin, we found a decreased risk for some cancers and treatment durations. However, the results for the other exposure categories showed no persistent differences even though an association between the increased cancer risk and use of insulin glargine would have been expected for the hypothesised effect. One possible explanation is that the better glycaemic control associated with use of insulin glargine, rather than human insulin [44], may play a part. So far, the evidence from epidemiological studies on the link between cancer risk and hyperglycaemia has been conflicting [45-48]. Similarly, increased site-specific cancer risks among individuals with type 1 and 2 diabetes, though showing a smaller excess risk for type 1 diabetes, suggest that a common diabetes-related determinant other than insulin use affects the cancer incidence [5]. Thus, to enhance knowledge on the interplay between diabetes and cancer, future research should focus on the effect of long-term glycaemic control itself, rather than different diabetes treatments.

This study has several strengths. To our knowledge, the present study is the first to employ a semi-aggregate level analysis across multiple populations from different countries to compare the effect of different insulin treatments on cancer risk. A semi-aggregate approach allowed us to analyse the study cohorts together using uniform methods. The size of the resulting cohort was fivefold that of the largest new-user cohort previously studied [40]. Our study had enough statistical power for the assessment of both sex- and site-specific cancer outcomes by cumulative durations; this assessment is considered by Renehan [16] to be an essential feature of an appropriately conducted pharmaco-epidemiological study on the link between insulin analogues and cancer risk.

Through the study design and analytical stages, we addressed important limitations and mitigated typical biases 
(Table 1) $[15,16,24,25,49]$. We adopted several other characteristics proposed by Renehan [16], including cohort design, use of validated data sources, exclusion of prevalent users and time-dependent definition of exposure. Moreover, we used an active-comparator design, which together with new-user design effectively reduces time-related biases and residual confounding $[15,25]$. In addition, rather than censoring at switching or stopping an insulin, we followed diabetic individuals through the entire insulin prescribing span. The advantages listed above, along with use of nationwide (Nordic countries) and representative population-based (CPRD) cohorts, provide generalisable findings that can be directly applied to real-word decision making.

Our study does, however, have some limitations, including lack of information on important risk factors such as smoking, BMI, sedentary lifestyle, family history of cancer and diabetes duration, type, severity and comorbidities. Diabetes duration has been associated with changes in cancer risk $[4,49]$ and obesity was found to contribute to the increased risk of colorectal cancer among individuals with type 2 diabetes [50]. Studies comparing insulin glargine users with human insulin users found little evidence of confounding by diabetes duration, hospitalisations or cancer screening [40], or by BMI, smoking, income or $\mathrm{HbA}_{1 \mathrm{c}}$ levels [38, 43, 51]. In addition to the use of active comparators, which reduces residual confounding, we accounted for the duration of insulin-treated diabetes, which could be an effect modifier of the relationship between insulin glargine use and cancer risk [37, 43], and adjusted for baseline use of non-insulin glucose-lowering therapy which can be considered as a proxy for one's diabetes stage when starting insulin treatment. We also performed sensitivity analyses to evaluate the effect of diabetes type.

Other disadvantages include lack of information on insulin dosage and the fact that exposure to non-insulin glucose-lowering therapy was defined as baseline usage without distinction between different therapies. Although we accounted for country-specific characteristics, we could not rule out any potential confounding effects resulting from the differences in insulin user profiles between the countries. In addition, examining numerous potential associations is likely to produce some false-positive results.

To summarise, the present multi-country study addressed the clinically important question of whether some of the commonly used insulin treatments should be preferred over others as being safer with respect to cancer risk. We found no persistent differences in the risk for ten specific cancers and any cancer, when comparing use of the insulin analogues insulin glargine or insulin detemir vs human insulin. These results add to the conclusive evidence on the absence of a relationship between cancer incidence and use of insulin analogues at follow-up exceeding 5 years. We see no indications to warrant withholding of the use of the insulin analogues insulin glargine and insulin detemir.
Data availability No additional data are available.

Funding This study was funded by the European Community's Seventh Framework Programme (FP-7) under grant agreement number 282526, the CARING project, and by the Finnish Cultural Foundation grant number 00160146. The funding sources had no role in the study design, data collection, data analysis, data interpretation or writing of the report.

Duality of interest AB's employment at the University of Helsinki was funded by the CARING project and Finnish Cultural Foundation. MTB's employment at Utrecht University was funded by the CARING project. MLDB is appointed as professor in Regulatory Science, which chair is funded by the University of Copenhagen. In addition, she is director of the Copenhagen Institute of Regulatory Science (CORS), based at the same university. CORS is a cross-faculty university anchored institution involving various public (Danish Medicines Agency, Copenhagen University) and private stakeholders (Novo Nordisk, Lundbeck, Ferring Pharmaceuticals, LEO Pharma) as well as patient organisations (Rare Diseases Denmark). The centre is purely devoted to the scientific aspects of the regulatory field and with a patient-oriented focus, and the research is not company-specific, product-specific or directly company related.

Apart from the position at the University of Copenhagen, MLDB is part-time employed by Utrecht University as a senior researcher conducting research under the umbrella of the Utrecht-WHO Collaborating Centre for Pharmaceutical Policy and Regulation. This centre receives no direct funding or donations from private parties, including the pharmaceutical industry. Research funding from public-private partnerships (e.g. Innovative Medicines Initiative [IMI]), The Escher Project (http://escher.lygature.org/) is accepted under the condition that no company-specific product or company-related study is conducted. The centre has received unrestricted research funding from public sources, e.g. the WHO, the Netherlands Organisation for Health Research and Development (ZonMW), the Dutch National Health Care Institute (ZIN), the EU 7th Framework Program (FP7), the Dutch Medicines Evaluation Board (MEB), and the Dutch Ministry of Health.

MA was employed by the Karolinska Institutet, the Research Unit For General Practice and by the University of Copenhagen supported by a grant from the Novo Nordisk Foundation (NNF15SA0018404), and participates/previously participated in projects funded by AstraZeneca, Pfizer, Novartis, Merck Sharp \& Dohme, H. Lundbeck, Mertz and Nycomed and received personal fees for leading and teaching pharmacoepidemiology courses at the Medicademy education programme, the Danish Association for the Pharmaceutical Industry. AA received lecture (MSD) and consulting (Epid Research Inc.) fees unrelated to the submitted work. FdV conducted research coordinated by The centre for Research Methods, which has received unrestricted funding from ZonMW, the Dutch Health Care Insurance Board (CVZ), the Royal Dutch Pharmacists Association (KNMP), the private-publicfunded Top Institute Pharma, the EU IMI, the EU FP7 and the Dutch Ministry of Health and industry (including GlaxoSmithKline, Pfizer and others). NE has served as a lecturer and on an advisory board for Astra Zeneca and Merck Sharp \& Dohme. JH is a Chief Science Officer and partner in Epid Research, which provides commissioned research services to industry. All other authors declare that there is no duality of interest associated with their contribution to this manuscript.

Contribution statement All authors made substantial contributions to the conception and design of the various aspects of this study or to acquisition, analysis or interpretation of data. All authors also contributed to drafting the article or revising it critically for important intellectual content and have given final approval of the version to be published. $A B$ is the guarantor of this study. 
Open Access This article is distributed under the terms of the Creative Commons Attribution 4.0 International License (http:// creativecommons.org/licenses/by/4.0/), which permits unrestricted use, distribution, and reproduction in any medium, provided you give appropriate credit to the original author(s) and the source, provide a link to the Creative Commons license, and indicate if changes were made.

\section{References}

1. Ferlay J, Steliarova-Foucher E, Lortet-Tieulent J et al (2013) Cancer incidence and mortality patterns in Europe: estimates for 40 countries in 2012. Eur J Cancer 49:1374-1403

2. International Diabetes Federation (2015) Diabetes atlas.7th edn. Available from www.diabetesatlas.org/, accessed 5 May 2017

3. Starup-Linde J, Karlstad O, Eriksen SA et al (2013) CARING (CAncer Risk and INsulin analoGues): the association of diabetes mellitus and cancer risk with focus on possible determinants - a systematic review and a meta-analysis. Curr Drug Saf 8:296-332

4. Harding JL, Shaw JE, Peeters A et al (2015) Cancer risk among people with type 1 and type 2 diabetes: disentangling true associations, detection bias, and reverse causation. Diabetes Care 38:264-270

5. Carstensen B, Read SH, Friis S et al (2016) Cancer incidence in persons with type 1 diabetes: a five-country study of 9,000 cancers in type 1 diabetic individuals. Diabetologia 59:980-988

6. Giovannucci E, Harlan DM, Archer MC et al (2010) Diabetes and cancer: a consensus report. Diabetes Care 33:1674-1685

7. Colhoun HM (2009) Use of insulin glargine and cancer incidence in Scotland: a study from the Scottish diabetes research network epidemiology group. Diabetologia 52:1755-1765

8. Currie CJ, Poole CD, Gale EAM (2009) The influence of glucoselowering therapies on cancer risk in type 2 diabetes. Diabetologia 52:1766-1777

9. Hemkens LG, Grouven U, Bender R et al (2009) Risk of malignancies in patients with diabetes treated with human insulin or insulin analogues: a cohort study. Diabetologia 52:1732-1744

10. Jonasson JM, Ljung R, Talbäck M et al (2009) Insulin glargine use and short-term incidence of malignancies-a population-based follow-up study in Sweden. Diabetologia 52:1745-1754

11. Pocock SJ, Smeeth L (2009) Insulin glargine and malignancy: an unwarranted alarm. Lancet 374:511-513

12. Baricevic I, Jones DR, Roberts DL et al (2015) A framework for the in vitro evaluation of cancer-relevant molecular characteristics and mitogenic potency of insulin analogues. Carcinogenesis 36:10401050

13. Gale EAM (2009) Insulin glargine and cancer: another side to the story? Lancet 374:521

14. Smith U, Gale EAM (2009) Does diabetes therapy influence the risk of cancer? Diabetologia 52:1699-1708

15. Wu JW, Filion KB, Azoulay L et al (2016) The effect of long-acting insulin analogs on the risk of cancer: a systematic review of observational studies. Diabetes Care 39:486-494

16. Renehan AG (2012) Insulin analogues and cancer risk: the emergence of second-generation studies. Diabetologia 55:7-9

17. Karlstad O, Starup-Linde J, Vestergaard P et al (2013) Use of insulin and insulin analogs and risk of cancer-systematic review and meta-analysis of observational studies. Curr Drug Saf 8:333-348

18. Badrick E, Renehan AG (2014) Diabetes and cancer: 5 years into the recent controversy. Eur J Cancer 50:2119-2125

19. Walker JJ, Johnson JA, Wild SH (2013) Diabetes treatments and cancer risk: the importance of considering aspects of drug exposure. Lancet Diabetes Endocrinol 1:132-139
20. Bordeleau L, Yakubovich N, Dagenais GR et al (2014) The association of basal insulin glargine and/or n-3 fatty acids with incident cancers in patients with dysglycemia. Diabetes Care 37:1360-1366

21. Zanders MMJ, Renehan AG, Bowker SL et al (2014) Comment on Bordeleau et al. The association of basal insulin glargine and/or $n-3$ fatty acids with incident cancers in patients with dysglycemia. Diabetes Care 37:1360-1366

22. CARING Consortium (2015) Common study protocol version 3.3. Available from www.encepp.eu/encepp/openAttachment/ fullProtocolLatest/9631, accessed 21 February 2017

23. Delgado-Rodriguez M, Llorca J (2004) Bias. J Epidemiol Community Health 58:635-641

24. Suissa S, Azoulay L (2012) Metformin and the risk of cancer: timerelated biases in observational studies. Diabetes Care 35:26652673

25. Yoshida K, Solomon DH, Kim SC (2015) Active-comparator design and new-user design in observational studies. Nat Rev Rheumatol 11:437-441

26. Engholm G, Ferlay J, Christensen N et al (2010) NORDCAN-a Nordic tool for cancer information, planning, quality control and research. Acta Oncol 49:725-736

27. Furu K, Wettermark B, Andersen M et al (2010) The Nordic countries as a cohort for pharmacoepidemiological research. Basic Clin Pharmacol Toxicol 106:86-94

28. Boggon R, van Staa TP, Chapman M et al (2013) Cancer recording and mortality in the general practice research database and linked cancer registries. Pharmacoepidemiol Drug Saf 22:168-175

29. Herrett E, Gallagher AM, Bhaskaran K et al (2015) Data resource profile: Clinical Practice Research Datalink (CPRD). Int J Epidemiol 44:827-836

30. WHO Collaborating Centre for Drug Statistics Methodology (2017) ATC/DDD Index 2017. Available from www.whocc.no/ atc_ddd_index/, accessed 20 March 2017

31. Bazelier MT, Eriksson I, de Vries F et al (2015) Data management and data analysis techniques in pharmacoepidemiological studies using a pre-planned multi-database approach: a systematic literature review. Pharmacoepidemiol Drug Saf 24:897-905

32. European Medicines Agency (2017). Lantus. Available from www. ema.europa.eu/ema/index.jsp?curl=pages/medicines/human/ medicines/000284/human_med_000882.jsp\&mid= WC0b01ac058001d124, last updated on 1 Mar 2017, accessed 5 May 2017

33. European Medicines Agency (2017). Levemir. Available from www.ema.europa.eu/ema/index.jsp?curl=pages/medicines/human/ medicines/000528/human_med_000884.jsp\&mid= WC0b01ac058001d124, last updated on $\overline{6}$ Feb 2017, accessed 30 Jan 2017

34. R Core Team (2015) R: a language and environment for statistical computing. R Foundation for Statistical Computing, Vienna www. R-project.org

35. Carstensen B, Plummer M, Laara E, Hills M (2015) Epi: a package for statistical analysis in epidemiology. R package version 1.1.71. Available from http://CRAN.R-project.org/package=Epi, accessed 21 February 2017

36. Gordon M, Lumley T (2017) Forestplot: advanced forest plot using grid graphics. R package version 1.7. Available from http:// CRAN.R-project.org/package=forestplot, accessed 21 February 2017

37. Suissa S, Azoulay L, Dell'Aniello S et al (2011) Long-term effects of insulin glargine on the risk of breast cancer. Diabetologia 54: 2254-2262

38. Stürmer T, Marquis MA, Zhou H et al (2013) Cancer incidence among those initiating insulin therapy with glargine versus human NPH insulin. Diabetes Care 36:3517-3525 
39. Ruiter R, Visser LE, van Herk-Sukel MPP et al (2012) Risk of cancer in patients on insulin glargine and other insulin analogues in comparison with those on human insulin: results from a large population-based follow-up study. Diabetologia 55:51-62

40. Fagot J-P, Blotière P-O, Ricordeau P et al (2013) Does insulin glargine increase the risk of cancer compared with other basal insulins? Diabetes Care 36:294-301

41. Gerhard T (2008) Bias: considerations for research practice. Am J Health Syst Pharm 65:2159-2168

42. NICE (2015) Type 2 diabetes in adults: management. Available from www.nice.org.uk/guidance/ng28, accessed 1 September 2016

43. Peeters PJHL, Bazelier MT, Leufkens HGM et al (2016) Insulin glargine use and breast cancer risk: associations with cumulative exposure. Acta Oncol 55:851-858

44. Fiesselmann A, Wiesner T, Fleischmann H, Bramlage P (2016) Real-world therapeutic benefits of patients on insulin glargine versus NPH insulin. Acta Diabetol 53:717-726

45. Saydah SH, Platz EA, Rifai N et al (2003) Association of markers of insulin and glucose control with subsequent colorectal cancer risk. Cancer Epidemiol Biomark Prev 12:412-418
46. Onitilo AA, Stankowski RV, Berg RL et al (2014) Type 2 diabetes mellitus, glycemic control, and cancer risk. Eur J Cancer Prev 23: 134-140

47. Johnson JA, Bowker SL (2011) Intensive glycaemic control and cancer risk in type 2 diabetes: a meta-analysis of major trials. Diabetologia 54:25-31

48. de Beer JC, Liebenberg L (2014) Does cancer risk increase with $\mathrm{HbA}_{1 \mathrm{c}}$, independent of diabetes? Br J Cancer 110:2361-2368

49. Johnson JA, Carstensen B, Witte D et al (2012) Diabetes and cancer (1): evaluating the temporal relationship between type 2 diabetes and cancer incidence. Diabetologia 55:1607-1618

50. Peeters PJHL, Bazelier MT, Leufkens HGM et al (2014) The risk of colorectal cancer in patients with type 2 diabetes: associations with treatment stage and obesity. Diabetes Care 38:495-502

51. Habel LA, Danforth KN, Quesenberry CP et al (2013) Cohort study of insulin glargine and risk of breast, prostate, and colorectal cancer among patients with diabetes. Diabetes Care 36:3953-3960 